\title{
EAl Endorsed Transactions

\section{Feature extraction of dance movement based on deep learning and deformable part model}

\author{
Shuang $\mathrm{Gao}^{1}$ and Xiaowei Wang ${ }^{2, *}$ \\ ${ }^{1}$ College of Music, Anhui Normal University, Wuhu, 241000, Anhui, China \\ ${ }^{2}$ Software College, Shenyang Normal University, Shenyang 110034, China \\ Email:407077764qq.com;zxcvfdsa5024@foxmail.com
}

\begin{abstract}
In complex scenes, the accuracy of dance movement recognition is not hi 1 . $\mathrm{Th}^{r}$ ffore, as paper proposes a deep learning and deformable part model (DPM) for dance movement feature ex ${ }^{+} \mathrm{act}_{\star}$ 、 1 rstly. he number of filters in DPM is increased, and the branch and bound algorithm is combined to impr t the ac rac secondly, deep neural network model is used to sample points of interest according to human danc mo. ments. '1. e features extracted from the DPM and deep neural network are fused. It achieves a large reductim in the $n_{\iota}$ vber of model parameters and avoids the network being too deep. Finally, dance movement recognition s performed on the input data through the full connection layer. Experimental results show that the proposed method in his paper an get the recognition result more quickly and accurately on the dance movement data set.
\end{abstract}

Keywords: DPM, dance movement feature extraction, deep no ral $\mathrm{y} / \mathrm{s}$ model.

Received on 23 December 2021, accepted on 31 D emb 2021, ublished on 05 January 2022

Copyright (C) 2022 Shuang Gao et al., licenond E E Crms an open access article distributed under the terms of the Creative Commons Attribution license, which perm unlimi use, distribution and reproduction in any medium so long as the original work is properly cited.

doi: 10.4108/eai.5-1-2022.172783

"Corresponding author. Ev 1: zxcy sa5024@ xmail.com

\section{Introduction}

Human motion recognition is a promising research field in computer vision. It has important research value in monitoring system, intelligent home and virtual reality. Different from simple image recognition, human motion recognition is affected by many factors, such as chaotic background, different image acquisition equipment, insufficient categories of human motion database and so on $[1,2]$. At present, there are mainly traditional machine learning and deep learning methods for human motion recognition.

In traditional human action recognition methods, manual design features are widely used for recognition, such as HOG/HOF et al, [3]. In reference [4], a new method of motion recognition was designed, and the obtained behavior features were fed into a vector machine to train the model. However, the recognition accuracy of traditional methods in real scene data sets is not too high, and the recognition results are not good [5].

In recent years, deep learning has attracted much attention in the field of human motion recognition. Convolutional neural network $(\mathrm{CNN})$ is a commonly used model in deep learning [6,7], which realizes image recognition through convolutional pooling of input images. The AlexNet network in 2015 made the CNN structure deeper [8]. Inception V2 network [9] made the CNN wider. Reference [10] sends both the time of video data and image information into $\mathrm{CNN}$, but the effect of dual-stream network was not ideal, and consumed a lot of memory and training time. Reference [11] proposed a new 
projection strategy in order to improve the recognition accuracy of tiny actions, which projected images onto multiple Cartesian planes to retain more behavior information. In reference [12], CNN convolution kernel was transformed into $3 \mathrm{D}$, and time dimension was added into the convolution kernel, which improved accuracy and increases training difficulty. Reference [13] combined CNN with LSTM to improve the accuracy of recognition. Reference [14] proposed Deformable Convolurtional Networks (DCN), which had more flexible position deformation ability compared with ordinary convolutional networks. Reference [15] combined Deformable Part Model (DPM) with CNN to improve the accuracy of pedestrian detection. In reference [16], pooling layer was improved in order to learn the unstable convolution features in images, and the experimental verification results were good. Although convolutional neural network improved the accuracy of image recognition, it was easy to lose the early feature information in the process of human action recognition.

To sum up, this paper integrates the improved DPM for dance movement recognition on the basis of deformable convolutional neural network (DCN). It combines traditional machine learning with deep learning to improve the accuracy and detection speed of dance movement recognition.

\section{DCN}

Deformable convolutional neural netwo proposed to improve the adaptability convo tional neural network to geometric de rion thigh deformable convolution and deform able regio of interest pooling [17]. These two method are based on tre idea of further irregular migration the convolution kernel sampling position information in th process of convolution pooling. $O$ sets re ob $\mathrm{d}$ by adding new modules without ada ional me deformable convolution is the ordin convolution. The deformable convolution is to add an on to the sampling position of the ordinary convolution. Deformable pooling is the addition of an offset for each bin location of the candidate region of interest. The offset is obtained by an additional layer of full connection. After the addition of modules, the deformable convolutional neural network can still be trained using the standard back propagation algorithm.

The comparison between ordinary convolution and deformable convolution is shown in figure 1 . Where, the size of the convolution kernel is $3 \times 3$. Figure 1 (a) shows ordinary convolution. The $3 \times 3$ convolution kernel is a rectangle of 9 points. Figure 1(b) and figure 1(c) represent deformable convolution. It adds an offset (arrow) to figure 1(a). Figure 1(b) shows the addition of offsets (arrows pointing to points) to the deformable convolution. Figure 1(c) shows the special case where a deformable convolution can reverse an angle.
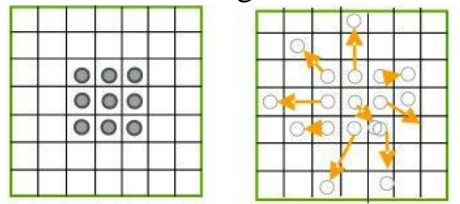

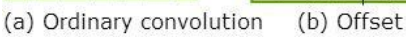

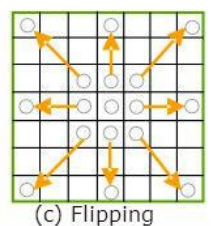

Figure 1. Tradition convo tion and deformable

3. DPM

Defo ab part mo (DPM) is an identification and detection mo od based on human body parts [18]. The model DPM forrecognizing human motion is composed of root ilter, component filter and relative position etween ilters. In general, the root filter has a lower res on and the component filter has a higher olution. Assuming that each target specifies the position of each filter in the model in the feature pyramid, $z=\left(t_{0}, \cdots, t_{m}\right)$ represents the DPM recognition model. Where, $m$ is the number of filters, which can be set by itself. $t_{i}=\left(x_{i}, y_{i}, l_{i}\right)$ represents the coordinates $\left(x_{i}, y_{i}\right)$ and the number of layers $l$ of the position of the i-th filter.

The score of the total test window is shown in equation (1):

$$
\begin{aligned}
& \operatorname{score}\left(t_{0}, \cdots, t_{m}\right)=\sum_{i=1}^{m} F_{i}^{\prime} \times \phi\left(H, t_{i}\right) \\
& -\sum_{i=1}^{m} d_{i} \times \phi_{d}\left(d x_{i}, d y_{i}\right)+b
\end{aligned}
$$

Where

$$
\left(d x_{i}, d y_{i}\right)=\left(x_{i}, y_{i}\right)-\left(2\left(x_{0}, y_{0}\right)+v_{i}\right)
$$

$\sum_{i=1}^{n} F_{i}^{\prime}$ represents the fractional sum of all the filters. $\phi\left(H, t_{i}\right)$ is the position of each component filter relative to the feature pyramid. $d_{i}$ is the offset loss coefficient. $\phi_{d}\left(d x_{i}, d y_{i}\right)$ represents the offset loss score of the i-th component filter. $b$ and $v_{i}$ represent different offset real 
numbers. $\left(x_{i}, y_{i}\right)$ is the spatial position of the filter response maximum value of the $\mathrm{i}$-th component. $\left(x_{0}, y_{0}\right)$ represents the coordinates of the layer where the root filter is located. $2\left(x_{0}, y_{0}\right)$ means that the resolution of the component filter is twice that of the root filter.

\section{Proposed feature extraction}

Traditional convolution operations use convolution kernels with fixed shapes, generally rectangular. Deformable convolution adds an offset for each convolution sampling point so that the convolution window convolves according to the region of interest. The window shape of standard convolution for human body recognition is regular rectangle, while the window shape of deformable convolution network can be changed according to the object instance. Taking the twodimensional convolution with a convolution kernel of $3 \times 3$ as an example, $M$ represents the effective receptive field:

$$
M=\{(-1,-1),(-1,0), \cdots,(0,-1),(1,1)\}
$$

After sampling on the input feature graph $x$, the offset is added to each sampling point and multiplied by the weight $w$. For the position $P_{0}$ on the output feature graph, the deformable convolution is expressed as:

$$
y\left(P_{0}\right)=\sum_{P_{n} \in M}\left(w\left(P_{n}\right) \cdot x\left(P_{0}+P_{n}+\Delta P_{n}\right)\right)
$$

Where, the offset parameter $\left\{\Delta P_{n} \mid\right.$

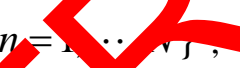
$N=|M|$ represents any position in,$P_{n}$ only as a certain influence on the input laye pixels, it does not affect the weight $w$, so botb $v$ and $\Delta P_{n}$ noed to be trained.

On the basis of ord ary conv utic, a convolution layer is added to ain $\mathrm{y}^{\mathrm{t}}$ offset, which is $\Delta P_{n}$ in equation (4). The offsen enerated has two directions of horizontal and vertical coor nates.

First, an increased convolution layer (conv) is used to obtain the offset of deformable convolution. Then the offset is shifted in the convolution kernel to complete the deformable convolution. Where $x$ is the input feature graph, and $y$ is the output feature graph. Interest pooling divides feature graphs into $\mathrm{k} \times \mathrm{k}$ bins $(\mathrm{k}=7$ is a freely set parameter).

In bin, deformable pooling of interest of row $i$ and column $j$ is expressed as:

$$
y(i, j)=\sum_{Q \in \operatorname{bin}(i, j)} \frac{x\left(Q_{0}+Q_{n}+\Delta Q_{i j}\right)}{n_{i j}}
$$

Where $Q_{0}$ is the point in the upper-left corner of bin. $Q_{n}$ represents any position in bin. $n_{i j}$ indicates the number of pixels in bin. $\Delta Q_{i j}$ is the offset. The structure of deformable pooling network is shown in figure 2 .

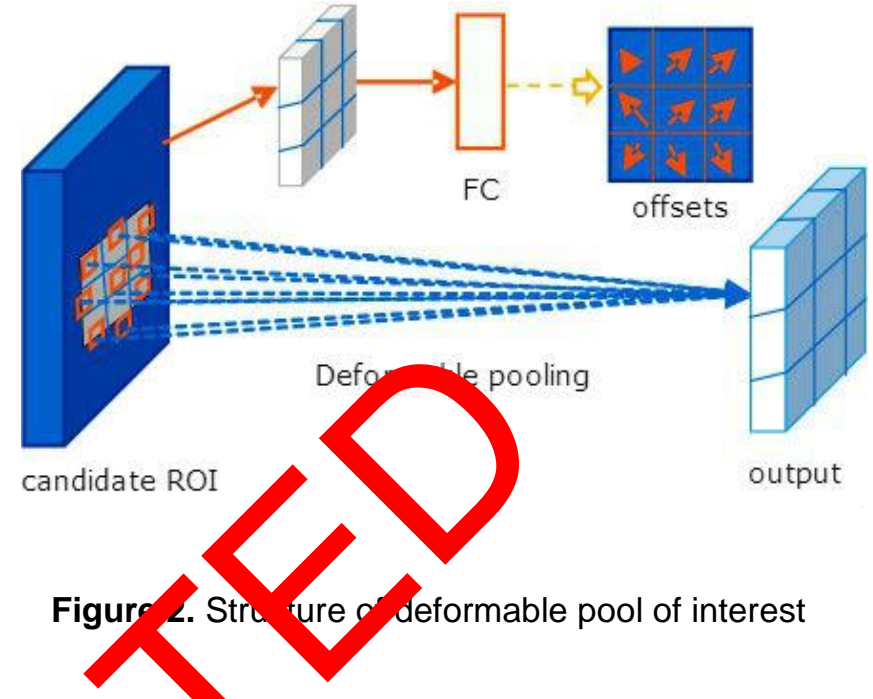

andidate in ions of interest (ROI) are obtained by pooling the feature map $[19,20]$. Then the offset of the region 9 interest is obtained through the new full nec on layer (FC). The input and output of the deformable convolutional network are the same as that of the ordinary convolutional network, except that during training, the newly added weights of the convolutional layer and the full connection layer for learning the offset are initialized to zero.

The deformable part is actually a model composed of a root filter and a component filter. The quality of DPM model is mainly determined by component filter. According to formula (1), $\sum_{i=1}^{n} F_{i}^{\prime}$ represents the total fraction sum of filters. Therefore, with the increase of the number of component filters, the calculation of $\sum_{i=1}^{n} F_{i}^{\prime}$ will become larger and the calculation speed will be correspondingly slower.

Experiments show that with the increase of component filters, although the detection accuracy is improved, the operation speed will be slowed down. In order to improve the experimental effect, we need to find a balance between the number of component filters and the operation speed. When the number of traditional DPM component models is 5 , the accuracy of the collected human motion data set is $83.75 \%$. When the number of component filters is increased to 8 , the accuracy reaches $95.67 \%$, and the accuracy of human motion recognition is improved by more than $11 \%$. However, when the number of filters reaches more than 8 , the improvement of accuracy is no longer obvious, which also brings some 
calculation difficulty to the experiment. Therefore, this paper increases the component models in DPM to 8.

DPM usually divides the human body into five parts in human movement recognition, namely head, left upper body, right upper body, legs and feet. In this paper, three models are added into the head, left shoulder, right shoulder, left abdomen, right abdomen, leg and left and right feet. The improvement point is that the original left upper body is further divided into left shoulder and left abdomen. The right upper body is divided into the right shoulder and the right abdomen. The feet are divided into left and right feet. The subdivided model can more accurately identify similar actions, such as running and playing football.

In order to solve the problem of excessive workload of DPM artificial design features, this paper integrates Branch and Bound (BB) algorithm [21] into DPM model to locate human body. Branch represents that the whole image is divided into several small regions, and the score value of each branch in the small region is calculated respectively. Bound is to set the optimal solution function boundary for each region and automatically find the maximum value in this region. The maximum region obtained by $\mathrm{BB}$ algorithm can be used as the region of interest for human movement recognition.

Traditional DPM recognition of human movements takes about 11s. After the BB algorithm is fused and the number of filters is increased to 8 , the detection speed of DPM is increased by about 3 times, and the resul ca be obtained in about 3.8s. According to the e erime tal data, traditional DPM takes a long time. $C$ mb with $\mathrm{BB}$ algorithm, it can quickly get the $\mathrm{w}$ imum ve of the function in the image and remo, $\mathrm{m}$, impossole hypothetical target actions, thus e ctively im oving the detection speed. The steps the nproved DPM to recognize human movements an allows

Step 1. Extracting featu

DPM uses directio al gre ient h, ogram (HOG) for feature extraction.

Step 2. DPM model.

Formula (1) is the sem ntic model of DPM, and modeling is to establish the structural model through the semantic model of formula (1). The DPM structural model consists of a root filter and several component filters. In this paper, experiments show that increasing the number of component filters from 5 to 8 can effectively improve the accuracy of human movement detection.

Step 3. DPM model training.

The root filter is initialized first. The region of interest determined by BB algorithm is scanned by root model, and the highest score is determined as the location of the root filter. The component filter is then initialized. The position of the component filter is determined according to the position relationship between the component filter and the root filter. After determining one component, the position of the next component is continued to be searched until the position of all component filters is determined. Finally, DPM is continuously updated until the accuracy was less than 0.01 .

Step 4 . The trained model is used to identify and classify the actions in the data set.

The following will introduce the depth feature extraction.

The proposed lightweight deep learning network that combines shallow and deep networks in this paper is shown in figure 3 . This network model mainly consists of two modules: one is shallow multi-scale network module, the other is deep network module. Specifically, the model in this paper consists of a convolution layer with a convolution kernel of $3 \times$ maximum pooling layer, three densely connect $a$ shallon multi-scale modules, a convolution layer with convolu on kernel of $1 \times 1 \times 1$, a deep network odule, ful connected layer and softmax. Der ry co rected rallow multi-scale modules are mainl espo ole for xtracting and combining local feature $f$ video $v$ ices to form longer and broader deep reatur The main function of deep network module better egrate the features extracted from the previous module by virtue of its better abstraction ability and enha ce the ability of the whole network model to press ne features. The combination of shallow and deep networks enables the model in this paper to better ro, resent the temporal and spatial features of video sequences and achieve better recognition results on the premise that the network model is not heavy enough for dance movement recognition tasks.

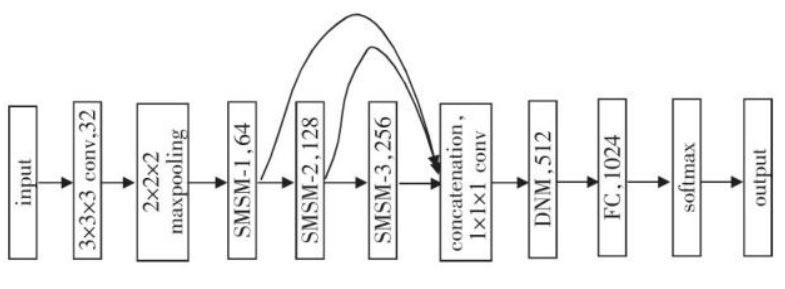

Figure 3. Lightweight deep learning network model combining shallow and deep networks, SDNet

\section{Shallow multiscale modules}

Generally speaking, motion feature extraction can be divided into global feature extraction and local feature extraction according to whether it is global feature extraction or not. However, for video-based human motion recognition tasks, most of the video background is complex environment or partial occlusion, and global feature extraction can not deal with this situation well. Multi-scale representation can be used to describe local features of video sequence in different scales in a simple way, which is convenient for analyzing local features of 
video frames. Therefore, this model adopts multi-scale feature extraction to extract local feature of video sequence.

Inspired by the RFB module [22], this paper adopts the operation of expansive convolution [23] to expand the acceptance domain of the network model and proposes the shallow multi-scale module (SMSM) based on the shallow network, as shown in figure 4 . Figure 4 takes the SMSM- 1 module in figure 3 as an example. The $1 \times 1 \times 1$ convolutional layer in this module does not contain nonlinear activation function, and the rest of the convolutional layer adopts ReLU as the nonlinear activation function. Specifically, this paper first uses $1 \times 1 \times 1$ convolution layer to construct a bottleneck structure for each branch of the module. The main purpose of this structure is to reduce the number of channels for the feature mapping of the input to the next layer, so as to improve the calculation efficiency. Then, two superimposed $3 \times 3 \times 3$ convolution layers are used to replace the $5 \times 5 \times 5$ convolution layer, which can effectively reduce model parameters. Finally, the features extracted from convolution layers with different expansion coefficients are connected to the next structure of the model through the maximum pooling layer. Human action recognition based on video often contain a longterm dependence of space and time, and the expansion of convolution operation has better application of this kind of problem, at the same time, the operation can be pooling operation and without loss of information and cep he same parameter, through increasing rece ive fild. enables the convolution operation to the la fer o ext or the output characteristic information. A re same ne, it has been proved in reference [24] th $/$ this peration can improve the calculation speed an recognitio accuracy. In this paper, two superposed $3 \times 3$ nvolutional layers in each branch of the SMSN a ale are inflated, as shown in figure 4, and th pans n co Aicients of each branch are respectivel set as 1,2,3\}. Meanwhile, in view of the expansion coes cie is sen $\{1,3,5\}$ in reference [25], this paper sets $u_{1}$ two groups of comparative experiments with different thansion coefficients in the experimental stage.

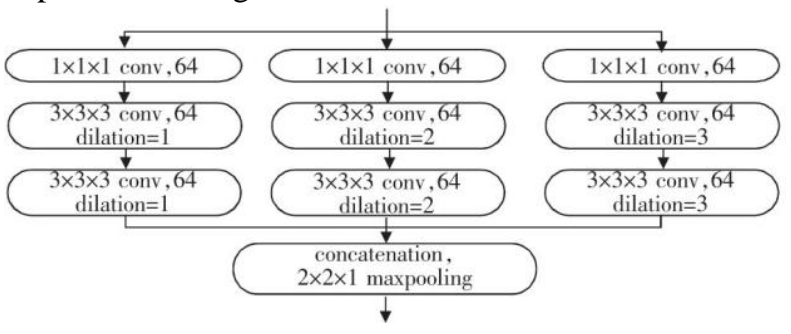

Figure 4. Shallow multi-scale module
Experiments in reference [26] had proved that densely connected networks not only did not bring redundancy problems, but also alleviated the problem of gradient disappearance and enhance the generalization ability of the network. The main reason is that the dense connection greatly reduces the computation amount of each layer, and the features extracted from each layer except the last layer of the dense connection can be reused. Therefore, it can be seen that dense connections can improve the performance of network models. Therefore, in this paper, features of three shallow multi-scale modules with different number of filters are densely connected to form longer and broader deep features. In order to better integrate the multi-scale features extracted from SMSM, this paper improved the module of deep network, and used the impr ed Net rk-In-Network (NIN) module to form therdee $\mathrm{R}_{\mathrm{N}}$ etwork odule in this model.

NIN module is a high ley of abstraction, in terms of human act n rec ghition ask, NIN module can be in the low-le $\mathrm{cl}$ to es for the top of the model, can get as muc s possib ur ar the same action from different angles, and ale features remain unchanged, the ability to her leve of abstraction can enhance human action recognition model in this paper the expression ability of local cha acteristics. NIN in reference [27] is shown in rure 5 The network can be approximated as consisting of three superposed NIN modules and a global average posling layer. Each NIN module consists of a $3 \times 3$ convolution layer, one $1 \times 1$ convolution layer and a combination of multi-layer perceptrons (MLPConv), and one $3 \times 3$ convolution layer. Among them, MLPConv and other convolution layers all take ReLU as their activation function. MLPConv has a stronger ability to model the distribution of various potential concepts than the simple linear convolution layer, which can improve the feature expression of traditional CNN.

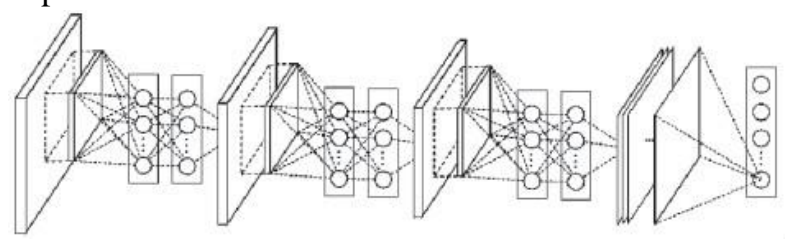

Figure 5. Overall structure of NIN

The deep network module of this article is shown in figure 6. NIN itself is superimposed by multiple CONV+MLPConv +CONV structures, and this paper adopts two such structures to superimpose to form deep network modules. Specifically, this paper firstly improves the original NIN in two aspects. On the one hand, considering the requirement of capturing spatio-temporal information as much as possible in the human motion recognition task in this paper, the convolution and pooling 
of each layer of the original NIN are extended to 3D operation. On the other hand, the effective fusion of multi-scale features after intensive connection of shallow multi-scale modules is considered due to the extensive application of receptive field operation for a large range of shallow multi-scale modules in SDNet. The first CONV+MLPConv+CONV structure of deep network module is improved. The expansion convolution operation with expansion coefficient of 2 is added to all convolution operations of the first structure. The first improved structure is then connected to the second improved structure through a maximum pooling layer of $2 \times 2 \times 2$. In the second improved structure, the convolution kernel expansion coefficient of all convolution is 1. Finally, considering that the global average pooling layer can reduce the number of parameters and make up for the shortcomings of the full connection layer, which is easy to over-fit, and meet the requirements of this paper to build a lightweight human action recognition model, the global average pooling method is still adopted in the classification layer as the feature mapping pooling method.

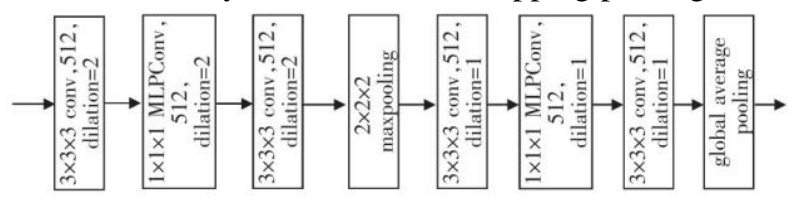

Figure 6. Deep networks module of SDNe+

\section{Feature fusion}

In this paper, the feature gra deformble convolutional neural network Ad DPM model is proposed to classify dance moyments. Although convolutional neural network s perior to traditional machine methods in dete spo and ccuracy, it has the problem of loy accu acy o low-level feature extraction. The fusio of roved DPM and the feature images extra ed from the deformable convolutional network be $\mathrm{ve}$ the deformable pooling layer can effectively improve the accuracy of low-level feature extraction of the convolutional neural network and thus improve the recognition accuracy of the global network. The pre-processed data sets are input into the deformable convolutional network and the improved DPM model respectively. The candidate regions of interest are obtained by the deformable convolutional network and the feature images are obtained by the fusion of the DPM. The fused feature images are used as the input of the deformable pooling of interest.

The dance movement recognition algorithm in this paper has four steps.

Step 1. Body parts are obtained using improved DPM. After the data set is preprocessed and input into the
network,DPM successively carries out the steps of feature extraction, modeling and training model. When DPM is used for human motion detection, BB algorithm can quickly get the optimal solution on the global graph, so that the DPM model can get the region of interest more quickly.

Step 2. Feature extraction from deformable convolutional networks. The same data set is used as input for the deformable parts model. First, three convolution blocks (including common convolution layer and pooling layer) are used to obtain low-level features. Then, the feature images are input into Region Proposal Network (RPN) $[28,29]$ through two layers of deformable convolution blocks (including ordinary convolution layer, deformable convolution layer and po layer) to obtain candidate region of interest. Us $\mathrm{g}$ back opagation algorithm to optimize parameters can lso prev ht over-fitting.

Step 3. Fusion $D$ DPM fe. ure vith candidate region of interest feat es. Te fracons of root filter and componer filte the or mal DPM model were added, that $\triangle_{i}^{n} F_{i}^{\prime}$ in aation (1) is obtained. Then feature $\Rightarrow \mathrm{F}$ is ob ined after a $3 \times 3$ convolution layer. Since deformable convolutional network is sampled layer by layer, up ampling operation of feature graph extracted by $\mathrm{NN}$ is cquired before feature fusion. $\mathrm{F}$ and up-sampled $R$ ruse features using weighted summation. The fused torure graph is used as the input graph of the deformable pooling layer. Finally, through the full connection layer, Softmax is used to judge the action category.

Step 4. Input the training set of the data set to train the model, and then input the data of the test set and validation set into the trained model to obtain the experimental results.

\section{Experiments and analysis}

\subsection{Experimental environment and data set}

In this paper, the hardware used to identify the system is NVIDIA GPU and the operating system is Windows 10. TensorFlow is combined with PyCharm to ensure the operation of the experiment. This paper selects two mainstream data sets in human motion recognition: MPII data set and MSCOCO Person Keypoints2017 data set (hereinafter referred to as MSCOCO data set). Both datasets are images collected in different scenarios that contain human movements in many complex situations, such as crowding, scale changes, occlusion and rotation. The annotated frames in the MPII dataset are divided into various action categories, such as running, skiing, and playing soccer, and the unannotated frames are used as the test set. A similar preprocessing method is also used 
for MSCOCO data sets, which commonly include speech, playing tennis, surfing and cooking in complex scenes. Finally, the robustness of the proposed method is proved by experiments in actual dance movements.

\subsection{Experimental training process and results}

In the forward propagation stage of the network, the training set is first used as the sample input into the designed model. The image is sampled layer by layer from input layer to output layer. In the backward propagation stage of the network, the weight is updated by calculating the error between the actual output image and the original image, and the process is repeated until the error is less than the preset value. The loss function of this experiment is the cross entropy function.

Accuracy rate is defined as:

$$
\text { acc }=\frac{\sum_{i=1}^{S} C_{i}}{S} \times 100 \%
$$

acc is the accuracy rate. The action category of the target in the dataset is $C_{i} . \mathrm{S}$ is the number of scenarios in the dataset

Table 1 and Table 2 are the experimental results on the MPII dataset and MSCOCO Person Keypoints2017 dataset respectively. As there are few methods combining traditional methods with $\mathrm{CNN}$, the convolution ne al network-based method with better performan in a recognition is mainly used in the comparat ex ext.

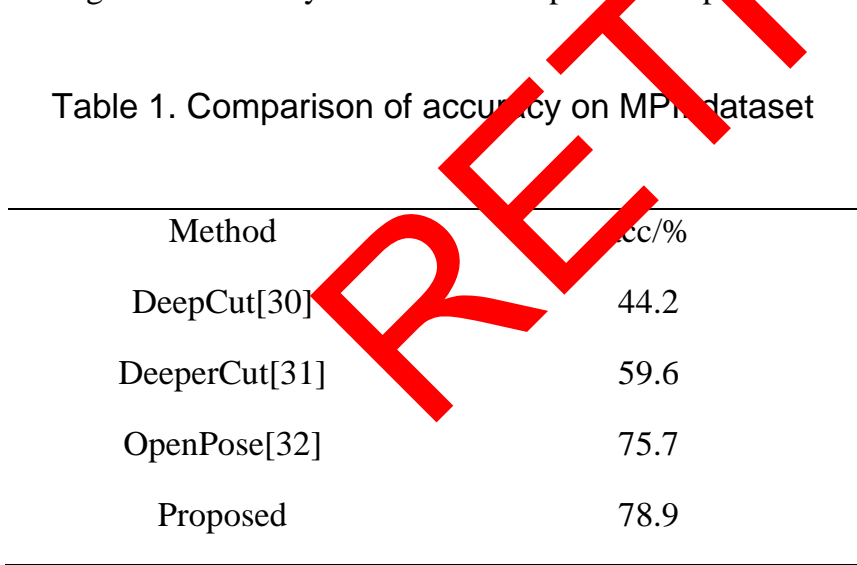

Table 2. Comparison of accuracy on MSCOCO dataset

$\begin{array}{cc}\text { Method } & \text { acc/\% } \\ \text { DeepCut[30] } & 61.9 \\ \text { DeeperCut[31] } & 64.1\end{array}$

\begin{tabular}{cc}
\hline OpenPose[32] & 66.6 \\
Proposed & 70.2
\end{tabular}

The recognition methods in reference [30,31] all recognize actions after human body is detected on ordinary convolution. The accuracy of recognition system in reference [31] is improved because CNN network becomes deeper. The recognition system in reference [32] analyzes the position of the human body after identifying the human body's key nodes using the ordinary convolutional network. In this paper, the method of reference [30] is also adopted, but deformable convolution is combined with improved DPM to identify human movements. It can be seen from the data in Table 1 that the identification netho in this paper has higher accuracy. In additio due to 1 combination of $\mathrm{BB}$ algorithm, the ca'culat speed has also been greatly improved. Un the sam perimental conditions, it takes $1.43 \mathrm{~s}$ fo con ron recognition system to process a single in ge, wh the cognition system in this paper only t $c$ bout 0.2

On MPIL ata set, confusion matrices of some action ategories are lected, as shown in figure 7. As can be seen fro figure 7 , the new method in this paper is elatively easy to identify actions with obvious c. istics, such as running and playing football. But there is a slight lack of recognition of complex movements, such as yoga and dancing, which are more confusing than running and playing football. Because there are so many kinds of yoga moves and they have a lot in common with dancing.

\begin{tabular}{|l|l|l|l|l|l|}
\hline & run & walk & football & dance & yoga \\
\hline run & 0.91 & 0.04 & 0.05 & 0.01 & 0.02 \\
\hline walk & 0.03 & 0.84 & 0.04 & 0.03 & 0.02 \\
\hline football & 0.03 & 0.04 & 0.92 & 0.01 & 0 \\
\hline dance & 0.03 & 0.05 & 0.01 & 0.85 & 0.05 \\
\hline yoga & 0.02 & 0.03 & 0.02 & 0.09 & 0.81 \\
\hline
\end{tabular}

Figure 7. Confusion matrix on MPII dataset

As can be seen from Table 2, the recognition system in this paper also has good experimental effect in complex scenes, with higher accuracy than the full convolutional network [32]. Although the recognition system in reference [31] adds tracking points on human body, the effect is not ideal.

Figure 8 is the confusion matrix of some action categories in the MSCOCO dataset. Most of the actions in 
MSCOCO are complex, so the accuracy is lower than that in MPII data set. As shown in figure 8, taking a selfie and making a phone call are generally similar hand-held mobile device actions, which are easy to confuse. Eating and drinking are generally sedentary activities, which are also prone to false detection. However, for actions with different postures, such as selfies and cooking, the false detection rate of the recognition method in this paper can be lower than $1 \%$ or even reach 0 .

\begin{tabular}{llllll}
\hline & selfie & cooking & call & drinking & eating \\
selfie & 0.84 & 0 & 0.14 & 0.01 & 0.02 \\
cooking & 0.01 & 0.74 & 0.02 & 0.08 & 0.14 \\
call & 0.11 & 0.02 & 0.81 & 0.03 & 0.01 \\
drinking & 0.01 & 0.05 & 0.03 & 0.76 & 0.11 \\
eating & 0.01 & 0.04 & 0.02 & 0.02 & 0.82 \\
\hline
\end{tabular}

Figure 8. Confusion matrix on MSCOCO dataset

\section{Conclusion}

In order to improve the accuracy of dance movemen recognition in complex scenes, this paper proposes an improved recognition system combining DPM learning. It adds the BB algorithm and th $\mathrm{DPM}$ to increase the dance movement recognition the amount of calculation is too large, aich me the detection speed slow down. The defe ria convo ion network model in the low-leyy featur extraction accuracy is low, so this arti combines aditional recognition algorithm, to is roy the $\mathrm{CNN}$ feature extraction accuracy. Howner, rece, sition method proposed in this paper all ha somo olems in practical application, such as clusi recognition accuracy, missing detection and to detection of complex actions. In the future, the hourg network model will be integrated into the deformable convolutional network, and the stacked hourglass network model will be used to improve the recognition accuracy of a single joint and further improve the accuracy of dance movement recognition.

\section{Acknowledgements.}

This work was supported by: Anhui Province Federation of Social Sciences, Researching Project "Overseas Broadcast of Dancing Art of Anhui Local Special Characteristics “(2121CX156).

\section{References}

[1] Liu, J., Rahmani, H., Akhtar, N. et al. Learning Human Pose Models from Synthesized Data for Robust RGB-D Action Recognition. Int J Comput Vis 127, 1545-1564 (2019). https://doi.org/10.1007/s11263-019-01192-2

[2] Dianhuai Shen, Xueying Jiang, Lin Teng. A novel GaussLaplace operator based on multi-scale convolution for dance motion image enhancement[J]. EAI Endorsed Transactions on Scalable Information Systems, 2021. http://dx.doi.org/10.4108/eai.17-12-2021.172439

[3] Nishimura K, Yanabe M, Nagatani T, et al. Proposal on Improvement of Defect Identification Performance by Dimensional Reduction Focusing on Localization of HOG Feature in Electronic Component Inspection[J]. Journal of the Japan Society for Precision Engineering, 2019, 85.

[4] S. Ramyar, A. Hom ar, A. arimoddini and E. Tunstel, "Identification of a malies in ne change behavior using one-class SVM " 201 IEEE Ir ernational Conference on Systems, M , and Cybo eti (SMC), 2016, pp. 004405004410, $10.1,9 /$ SMC. 016.7844924.

[5] $\mathrm{W}_{\mathrm{a}} \mathrm{X}, \mathrm{Go}, \mathrm{G}, \mathrm{N}$, et al. Decoding pilot behavior Iisa usness of G, ECG, eye movements via an SVM machino learning model[J]. International Journal of Modeling Simulation and Scientific Computing, 2020 (15):2050028.

Qin vu Shi, Shoulin Yin, Kun Wang, Lin Teng and Hang Multichannel convolutional neural network-based fuzzy active contour model for medical image segmentation. Evolving Systems (2021). https://doi.org/10.1007/s12530-021-09392-3

[7] Jisi A and Shoulin Yin. A New Feature Fusion Network for Student Behavior Recognition in Education [J]. Journal of Applied Science and Engineering. vol. 24, no. 2, pp.133-140, 2021.

[8] S. Gu, L. Ding, Y. Yang and X. Chen, "A new deep learning method based on AlexNet model and SSD model for tennis ball recognition," 2017 IEEE 10th International Workshop on Computational Intelligence and Applications (IWCIA), 2017, pp. 159-164, doi: 10.1109/IWCIA.2017.8203578.

[9] Baldassarre F, Morín, Diego González, Rodés-Guirao, Lucas. Deep Koalarization: Image Colorization using CNNs and Inception-ResNet-v2[J]. 2017. arXiv: 1712.03400

[10] Aslan M F, Durdu A, Sabanci K. Human action recognition with bag of visual words using different machine learning methods and hyperparameter optimization[J]. Neural Computing and Applications, 2020, 32(12):8585-8597.

[11] Afza F, Khan M A, Sharif M, et al. A framework of human action recognition using length control features fusion and Weighted Entropy-Variances based Feature Selection[J]. Image and Vision Computing, 2021, 106:1-20. 
[12] Indhumathi C, Murugan V, Muthulakshmii G. Human Action Recognition Using Spatio-Temporal Multiplier Network and Attentive Correlated Temporal Feature[J]. International Journal of Image and Graphics, 2021.

[13] S. Kumawat, M. Verma, Y. Nakashima and S. Raman, "Depthwise Spatio-Temporal STFT Convolutional Neural Networks for Human Action Recognition," in IEEE Transactions on Pattern Analysis and Machine Intelligence, doi: 10.1109/TPAMI.2021.3076522.

[14] Suresh A J, Visumathi J. Inception ResNet deep transfer learning model for human action recognition using LSTM - ScienceDirect[J]. Materials Today: Proceedings, 2020.

[15] J Lee, Ahn B. Real-Time Human Action Recognition with a Low-Cost RGB Camera and Mobile Robot Platform[J]. Sensors (Basel, Switzerland), 2020, 20(10).

[16] Jia J G, Zhou Y F, Hao X W, et al. Two-Stream Temporal Convolutional Networks for Skeleton-Based Human Action Recognition[J]. Journal of Computer Science and Technology, 2020, 35(3):538-550.

[17] Fei L, Dan L C, Jie T, et al. Cascaded one-shot deformable convolutional neural networks: Developing a deep learning model for respiratory motion estimation in ultrasound sequences[J]. Medical Image Analysis, 2020, 65.

[18] Dou J, Li J. Robust object detection based on deformable part model and improved scale invariant featur transform[J]. Optik - International Journal for Light and Electron Optics, 2013, 124(24):6485-6492.

[19] Shoulin Yin, Hang Li, Desheng Liu and $\mathrm{Sb}$ Id Ka $\mathrm{m}$. Active Contour Modal Based on Density Clustering Method for Medical Image cgmen. on [J]. Multimedia Tools and Applications $\sim 79$, pp. 49 31068, 2020.

[20] S. Yin and H. Li. Hot Regiop,election Based on Selective Search and Modified Fuzzy -M as in Remote Sensing Images[J]. IEEE Journ of $\mathrm{S}$ cted $\mathrm{T}$ pics in Applied Earth Observations a Re ote So i vol. 13, pp. 58625871, 2020, doi: 1109/ TARS.2020.3025582.

[21] Panja S, Mondal S K Analytics of an Imperfect FourLayer Production Inven ry Model Under Two-Level Credit Period Using Branch-and-Bound Technique[J]. Journal of the Operations Research Society of China, 2020(1).

[22] S. Hou, Y. Li, Y. Pan, X. Yang and G. Yin, "A Face Detection Algorithm Based on Two Information Flow Block and Retinal Receptive Field Block," in IEEE Access, vol. 8, pp. 30682-30691, 2020, doi: 10.1109/ACCESS.2020.2973071.

[23] Yin, S., Li, H. \& Teng, L. Airport Detection Based on Improved Faster RCNN in Large Scale Remote Sensing Images [J]. Sensing and Imaging, vol. 21, 2020. https://doi.org/10.1007/s11220-020-00314-2
[24] Xiaowei Wang, Shoulin Yin, Ke Sun, et al. GKFC-CNN: Modified Gaussian Kernel Fuzzy C-means and Convolutional Neural Network for Apple Segmentation and Recognition [J]. Journal of Applied Science and Engineering, vol. 23, no. 3, pp. 555-561, 2020.

[25] Li M, Sun Q. 3D Skeletal Human Action Recognition Using a CNN Fusion Model[J]. Mathematical Problems in Engineering, 2021, 2021.

[26] G. Huang, Z. Liu, L. Van Der Maaten and K. Q. Weinberger, "Densely Connected Convolutional Networks," 2017 IEEE Conference on Computer Vision and Pattern Recognition (CVPR), 2017, pp. 2261-2269, doi: 10.1109/CVPR.2017.243.

[27] Lin M, Chen Q, Yan S. Network In Network[J]. Computer Science, 2013.

[28] Shoulin Yin, Hang L Lin Teng et al. An optimised multiscale fusion nethod to airp $t$ detection in large-scale optical re ote ser ing ima s [J]. International Journal of Image nd rusion ol. 11, no. 2, pp. 201-214, 2020. DO 10.1080 ( 4799 2.2020.1727573

[29] Jing Hang Li, Shoulin Yin. Dynamic Gesture Recognitio Based on Deep Learning in Human-toComputer Interfaces $[\mathrm{J}]$. Journal of Applied Science and Eng eering, vol. 23, no. 1, pp.31-38, 2020.

U., Gall J. (2016) Multi-person Pose Estimation with Local Joint-to-Person Associations. In: Hua G., Jégou H. (eds) Computer Vision - ECCV 2016 Workshops. ECCV 2016. Lecture Notes in Computer Science, vol 9914, pp. 627-642. Springer, Cham. https://doi.org/10.1007/978-3319-48881-3_44

[31] Insafutdinov E., Pishchulin L., Andres B., Andriluka M., Schiele B. (2016) DeeperCut: A Deeper, Stronger, and Faster Multi-person Pose Estimation Model. In: Leibe B., Matas J., Sebe N., Welling M. (eds) Computer Vision ECCV 2016. ECCV 2016. Lecture Notes in Computer Science, vol 9910, pp. 34-50. Springer, Cham. https://doi.org/10.1007/978-3-319-46466-4_3

[32] Z. Cao, T. Simon, S. Wei and Y. Sheikh, "Realtime Multiperson 2D Pose Estimation Using Part Affinity Fields," 2017 IEEE Conference on Computer Vision and Pattern Recognition (CVPR), 2017, pp. 1302-1310, doi: 10.1109/CVPR.2017.143. 